\title{
Cooperative Sentry Vehicles And Differential GPS Leapfrog
}

\author{
John Feddema, Chris Lewis, Robert LaFarge \\ Intelligent Systems and Robotics Center \\ Sandia National Laboratories" \\ Albuquerque, NM 87185-1003
}

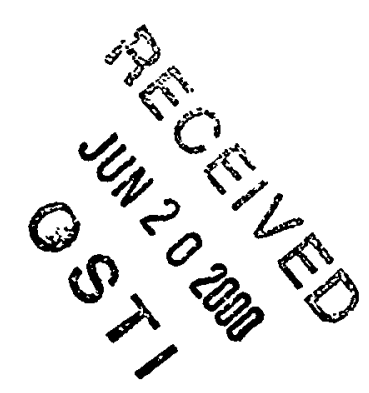

\begin{abstract}
As part of a project for the Defense Advanced Research Projects Agency, Sandia National Laboratories' Intelligent Systems \& Robotics Center is developing and testing the feasibility of using a cooperative team of robotic sentry vehicles to guard a perimeter, perform a surround task, and travel extended distances. This paper describes our most recent activities. In particular, this paper highlights the development of a Differential Global Positioning System (DGPS) leapfrog capability that allows two or more vehicles to alternate sending DGPS corrections. Using this leapfrog technique, this paper shows that a group of autonomous vehicles can travel 22.68 kilometers with a root mean square positioning error of only 5 meters.
\end{abstract}

Keywords: Mobile Robots, Cooperative Control, Distributed Autonomous Systems

\section{Introduction}

The field of mobile robotics is quite advanced. The ability to build robotic vehicles that can navigate over long distances either using tele-operation or autonomous control has been demonstrated by a number of researchers, see for instance [1]. In recent years, this field has expanded to consider large numbers or squads of vehicles [2]. The underlying goal of multi-vehicle systems is expanded capability through cooperation. Methods for controlling groups of vehicles range from distributed autonomy [3] to intelligent squad control and general purpose cooperative mission planning [4]. The types of tasks under study range from moving

\footnotetext{
- Sandia is a multiprogram laboratory operated by Sandia Corporation, a Lockheed Martin Company, for the United States Department of Energy under contract DEAC04-94AL85000. This research is partially funded by the Advanced Technology Office of the Defense Advanced Research Projects Agency under contract e169/09.
} 
large objects [5] to troop hunting behaviors [6]. Conceptually, large groups of mobile vehicles outfitted with sensors should be able to automatically perform military tasks like formation following, localization of chemical sources, demining, perimeter control, surveillance, and search and rescue missions [7-10]. Simulation has shown that by sharing concurrent sensory information, the group can better estimate the shape of a chemical plume and therefore localize its source [11]. Similarly, for a search and rescue operation, a moving target is more easily found using an organized team [12-13]. Simulation has also shown that enhanced perimeter control can be achieved by dispersing the group uniformly and by communicating when possible intrusions occur.

As a proof-of-concept, Sandia National Laboratories is developing a squad of semi-autonomous all terrain vehicles for remote cooperative sensing applications (see Figure 1). The system is being used to demonstrate the viability of using a cooperative team of robotic sentry vehicles to investigate alarms from intrusion detection sensors and to surround and monitor an enemy facility.

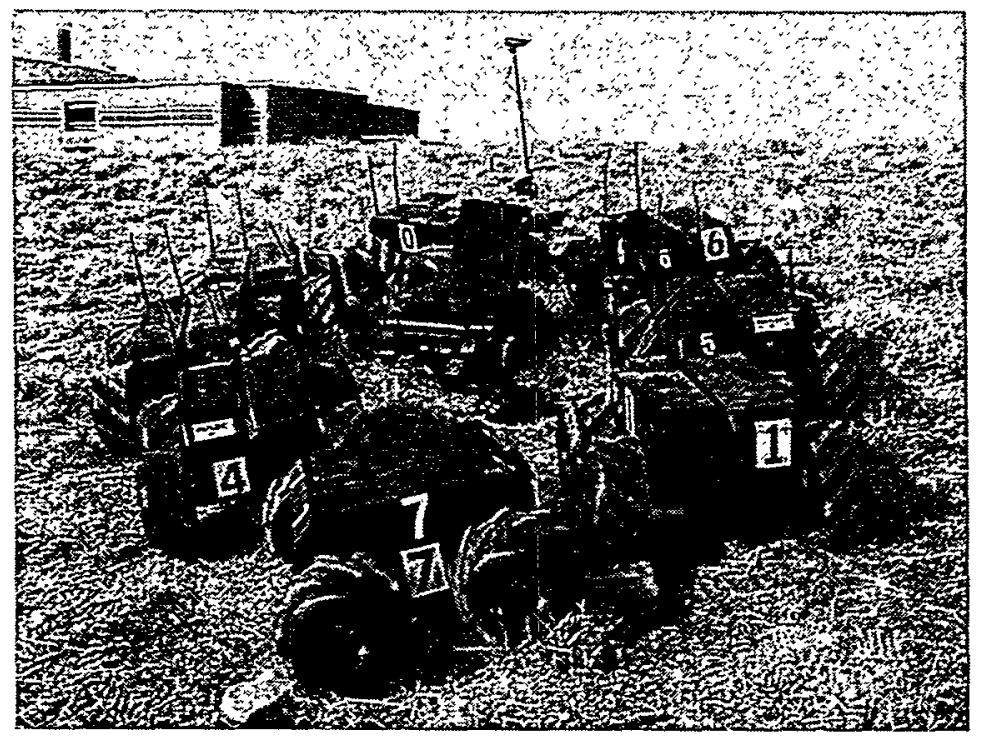

Figure 1. 8 RATLER vehicles around the laptop base-station.

To date, we have developed a robotic perimeter detection system which consists of eight "Roving All Terrain Lunar Explorer Rovers" (RATLERTM), a laptop-based base-station, and several Miniature Intrusion Detection Sensors (MIDS). A radio frequency receiver on each of the RATLER vehicles alerts the sentry vehicles of alarms from the hidden MIDS. When an alarm is received, each vehicle decides whether it should investigate the alarm depending on the proximity of itself and 


\section{DISCLAIMER}

This report was prepared as an account of work sponsored by an agency of the United States Government. Neither the United States Government nor any agency thereof, nor any of their employees, make any warranty, express or implied, or assumes any legal liability or responsibility for the accuracy, completeness, or usefulness of any information, apparatus, product, or process disclosed, or represents that its use would not infringe privately owned rights. Reference herein to any specific commercial product, process, or service by trade name, trademark, manufacturer, or otherwise does not necessarily constitute or imply its endorsement, recommendation, or favoring by the United States Government or any agency thereof. The views and opinions of authors expressed herein do not necessarily state or reflect those of the United States Government or any agency thereof. 


\section{DISCLAIMER}

Portions of this document may be illegible in electronic image products. Images are produced from the best available original document. 
the other vehicles to the alarm. As one vehicle attends an alarm, the other vehicles adjust their position around the perimeter to better prepare for another alarm [14].

For the surround task, both potential field and $\mathrm{A}^{*}$ search path planners have been added to the base-station and vehicles [15]. At the base-station, the operator specifies goal and exclusion regions on a GIS map. The path planner generates vehicle paths that are previewed by the operator. Once the operator has validated the path, the appropriate information is downloaded to the vehicles. On board the vehicles, the path planner generates the path except that it uses the true location of itself and the nearest neighboring vehicle to avoid collisions.

Most recently, we have added the capability for multiple robots to travel long distances using a differential GPS leapfrog technique. This paper first describes the system and the basic communication modes that have been implemented. This is followed by a detailed description of the differential GPS leapfrog technique.

\section{System Description}

Eight RATLER ${ }^{\mathrm{TM}}$ vehicles have been built at Sandia as a test platform for cooperative control and sensing applications. These electric, all wheel drive vehicles consist of two composite bodies joined by a passive central pivot. This flexible structure when combined with an aggressive asymmetric tread on custom carbon composite wheels provides agile off road capabilities. The RATLER vehicles are equipped with a PC104 form factor Intel 80486 processor for control. This computer interfaces to a wide range of sensors and peripherals. Software on the vehicles is currently a single-threaded DOS-based application for simplicity. The vehicles have been programmed to operate either through tele-operation or autonomously. The RATLER vehicles rely heavily on Radio Frequency (RF) signals for communications. Currently, the vehicles are outfitted with differential GPS sensors, and two spread spectrum RF modems. One modem is for inter-vehicle and base-to-vehicle communication and the other is for the differential GPS signal. Video cameras communicate to the base-station via a separate $\mathrm{RF}$ video link.

A laptop computer is used as the base-station. A Windows NT application was written to control the vehicles from the base-station. A Graphical User Interface (GUI) displays vehicle status information and allows the operator to monitor the vehicles positions on a Geographic Information System (GIS) map - either aerial photo or topological data, as well as view the live video from a selected vehicle. Mission specific control modes such as tele-operation, formation following, autonomous navigation, and perimeter detection can be initiated and monitored using this GUI interface. 
Star

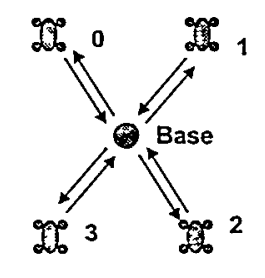

Basestation is single point of failure.
Token Ring

with Timeout

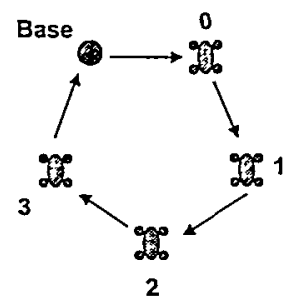

More fault tolerant. Allows basestation to be shutdown.

Figure 2 Two communication options are available. The star network requires the base-station to coordinate all communication. The token ring network provides a decentralized solution.

\section{Communication}

The RF communication network between the RATLER vehicles and the basestation can be configured as either a star or token ring (see Figure 2). With the star network, all communication is coordinated by the base-station. The base-station sends messages to each vehicle in turn, and each vehicle responses with a broadcast to all the network nodes (the base-station and all the other vehicles). With the token ring network, the base-station and vehicles each take turns broadcasting messages over the network. Each node (either vehicle or base-station) speaks only when it receives the token. The token ring network is more fault tolerant than the star network because there is no single point of failure as there is with the star network. Also, the token ring network allows the vehicles to continue operating even if the base-station is shutdown.

\section{Differential GPS Leapfrog}

In order to understand the differential GPS leapfrog capability, we must first review the basic concepts of the Differential Global Positioning System (DGPS). The standard GPS receiver measures the time of flight (or raw pseudorange $\rho$ ) of transmitted RF messages from four or more satellites [15]. This measured value is corrupted by noise. In general, the measured pseudorange is equal to the true range $\mathrm{D}$ from the user to the satellite plus an unknown offset between the user clock and the satellite clock. Additional time delays are caused by the ionosphere and troposphere, as well as noise multipath, and/or interchannel errors in the user's receiver. The corrected pseudorange is 


$$
\rho_{c}=\rho-c(\hat{I}+\hat{T}-\hat{B})
$$

where $\mathrm{c}$ is the speed of light; and $\hat{I}, \hat{T}$, and $\hat{B}$ are the estimate time delays caused by the ionosphere, troposphere, and satellite clock. Table 1 shows the estimated effects of these delays.

Table 1. Typical error in meters per satellite.

\begin{tabular}{|c|c|c|}
\hline & Standard GPS & Differential GPS \\
\hline Satellite Clocks & 1.5 & 0 \\
\hline Orbit Errors & 2.5 & 0 \\
\hline Ionosphere & 5.0 & 0.4 \\
\hline Troposphere & 0.5 & 0.2 \\
\hline Receiver Noise & 0.3 & 0.3 \\
\hline Multipath & 0.6 & 0.6 \\
\hline SA & 30 & 0 \\
\hline
\end{tabular}

Using a least squares solution, the estimated user position $\hat{\bar{r}}_{u}$ and user clock offset $\hat{b}_{u}$ is

$$
\hat{\vec{x}}=G^{+}\left(A \vec{R}-\vec{\rho}_{c}\right)
$$

where

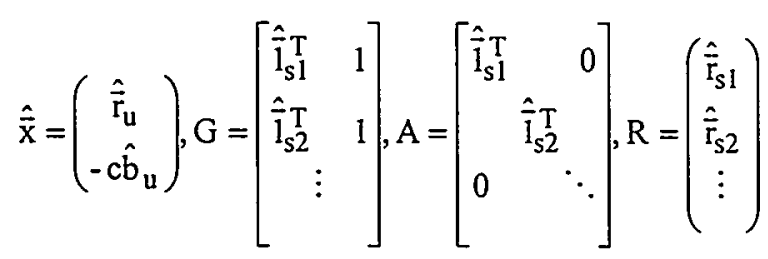

The matrices $G$ and $A$ are composed of the estimated directions $\hat{\bar{I}}_{s n}$ of each of the visible satellites. The vector $R$ is the estimated satellite positions that have been received as part of the satellite broadcast. The vector $\bar{p}_{c}$ is the corrected pseudorange to each satellite, arranged as a vector.

In a DGPS system, a reference receiver is put in a known location so that the corrections to the raw pseudorange can be found by:

$$
\Delta \hat{\vec{\rho}}_{R}=A \vec{R}-\bar{\rho}-G \vec{x}_{R}
$$

The reference receiver transmits this correction to all users. It is assumed that if the reference receiver and the user are near each other, then they both have the same pseudorange correction. As shown in Table 2, the horizontal position accuracy improves from 50 meters (without differential corrections) to approximately 
0.67 meters (with differential corrections). Even the military's Precision Positioning Service, with a normal positioning accuracy of 15 meters, is improved to less than 1 meter with DGPS corrections.

Table 2. Typical Position Accuracy.

\begin{tabular}{|l|l|l|}
\hline & Standard GPS & Differential GPS \\
\hline Horizontal & 50 & 0.67 \\
\hline Vertical & 78 & 2.0 \\
\hline 3-D & 93 & 2.8 \\
\hline
\end{tabular}

While performing coordinated robotic vehicle maneuvers such as formation following or perimeter control, it is important to be able to precisely locate each individual vehicle's absolute location. For this reason, DGPS is used on most robotic vehicle platforms for position control. However, the one downfall to DGPS is that it requires that one GPS receiver (typically at the base-station) be surveyed to a know location. Another alternative to a surveyed point is keep a receiver in a single position for a long period of time (as much as 24 hours), and use the average position as the "true" location when determining the differential correction.

Neither of these options is viable for most military operations where there are no surveyed points and there is no time to wait. For these situations, we have developed a DGPS leapfrog technique where one vehicle stops and becomes a base GPS unit while the others get corrections from it. The process is initialized by one GPS receiver (this could be the base-station or a vehicle) having a known location, either surveyed or time averaged. Corrections from this receiver are transmitted to the others. As the other vehicles reach the communication range of the first, another vehicle becomes the base GPS unit using the differentially corrected position from the first vehicle. This frees the first vehicle to again become a free roaming vehicle using differential corrections from the second. This process may be repeated indefinitely; however, eventually the accumulated error will be above an acceptable level. At which point, the newly assigned GPS base vehicle is required to stay in one position and average GPS measurements until the absolute positioning error is back to an acceptable level.

The question arises as to how many leapfrog steps are acceptable? The accuracy of the leapfrog technique may be analyzed using a random walk. Assume that each time two vehicles exchange roles as GPS base, the expected RMS positioning error of the second vehicle relative to the first is $r_{\text {rms. }}$. After $N$ leapfrog steps, the total expected RMS error is given by

$$
R^{2}=E\left\{\left(\Delta x_{1}+\ldots+\Delta x_{N}\right)^{2}+\left(\Delta y_{1}+\ldots+\Delta y_{N}\right)^{2}\right.
$$

where $\left(\Delta \mathrm{x}_{1}, \Delta \mathrm{y}_{1}\right) \ldots\left(\Delta \mathrm{x}_{\mathrm{N}}, \Delta \mathrm{y}_{\mathrm{N}}\right)$ are the errors in $\mathrm{x}$ and $\mathrm{y}$ positions. Assuming each of the $\mathrm{N}$ leapfrog steps is independent, this error reduces to

$$
R^{2}=E\left\{\Delta x_{1}^{2}+\Delta y_{1}^{2}\right\}+\ldots+E\left\{\Delta x_{N}^{2}+\Delta y_{N}^{2}\right\}=N r_{r m s}^{2} .
$$


Solving for the number of leapfrog steps,

$$
N=\left(R / r_{r m s}\right)^{2}
$$

Therefore, if the acceptable accumulated error of the robot vehicles is 5 meters, and the RMS error of each step is 0.67 meters, then two or more vehicles can travel 22.86 kilometers in 56 steps, each step being 0.4 kilometers long.

This DGPS leapfrog mode of operation has been installed on our RATLER test bed. Figure 3 shows the base-station's graphical user interface being used to direct two RATLER vehicles along a test path while a third, stationary vehicle monitors the accumulated error in the corrections. To run the test, the operator first draws a path for both vehicles. At intermediate waypoints, the operator alternates selecting each vehicle to be the GPS base-station (denoted by green triangles in the figure). Currently, the operator must be sure to select adjacent GPS base positions that are within range of each vehicle's radio that transmits and receives the GPS corrections. This process could be automated in the future.

To execute the test path, the waypoints and exchange point are downloaded to the robots over the communications link. Then, both vehicles are activated. Initially they both use the command station's GPS receiver for corrections. When the first vehicle reaches a waypoint node designated by the operator as a DGPS node, it stops, and uses its corrected GPS position to re-initialize its GPS receiver to act as a correction station. It also sends a command to the base station to turn off the base station GPS receiver. From there on, the base station is not necessary to the operation. The vehicles execute exchanges by sending commands to each other autonomously. For the test, a third motionless vehicle's position was monitored and recorded throughout the course. The accumulated change in its recorded position was expected to follow a random walk model.

The path including 12 exchanges is shown in Figure3. It was executed 5 times. The position data recorded from the stationary vehicle is shown in Figure 4 . The first test run accumulated an error of 2 meters, while each of the following 4 runs only accumulated a 1 meter error.

\section{Conclusion}

This paper described a squad of mobile robotic vehicles being developed at Sandia National Laboratories to demonstrate cooperative robotic sensing capabilities. The squad currently consists of eight RATLER ${ }^{\mathrm{TM}}$ vehicles, a laptop base-station, and a battery powered antenna array. In the past, this system has been used to demonstrate autonomous navigation of a cooperative team of robots and their use for surround and perimeter detection missions. This paper highlights the use of a 
DGPS leapfrog technique that allows a squad of vehicles to travel long distances without maintaining communication to a base-station

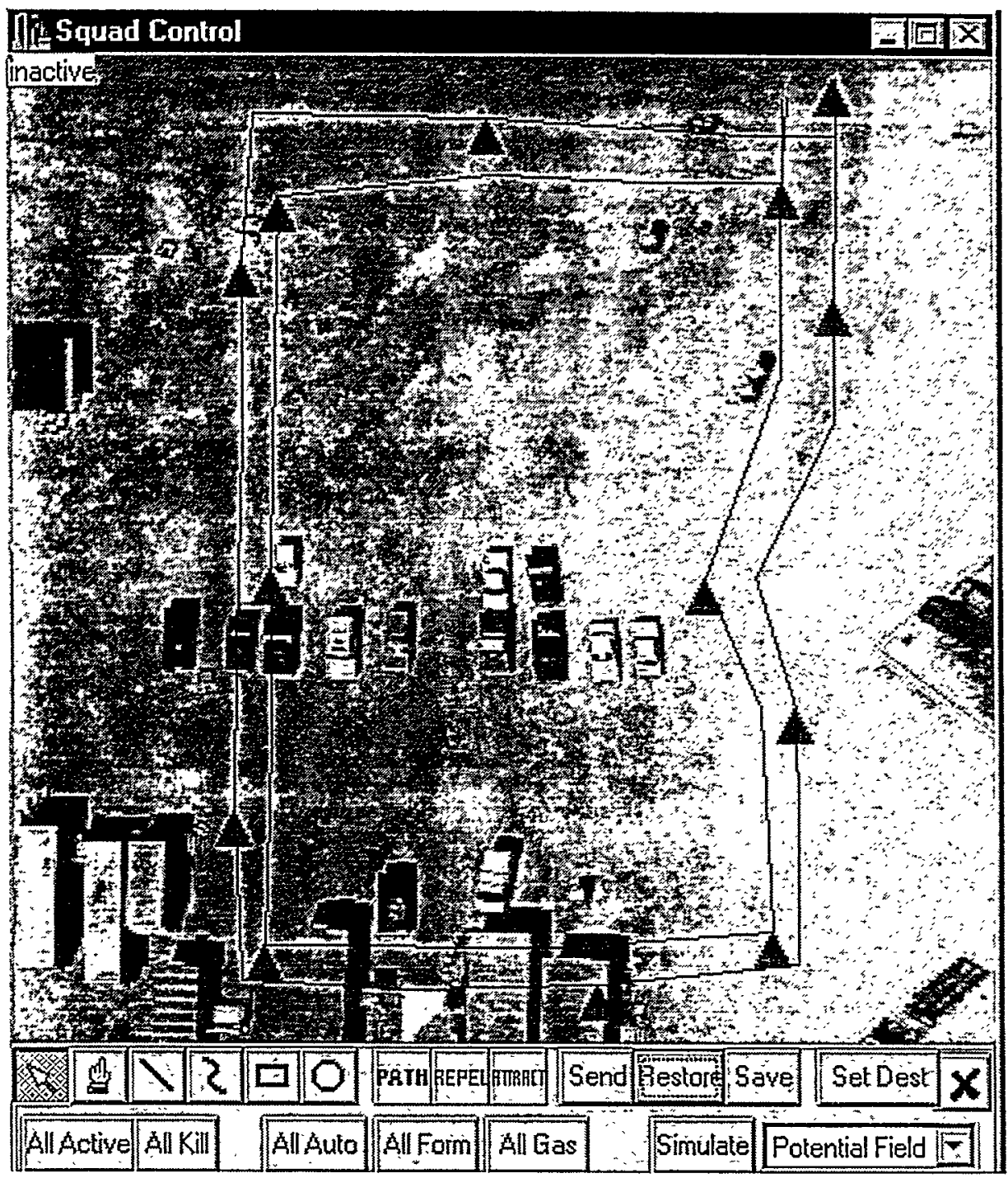

Figure 3 GUI used to designate paths and exchange points for two vehicles to demonstrate the DGPS leapfrog technique 


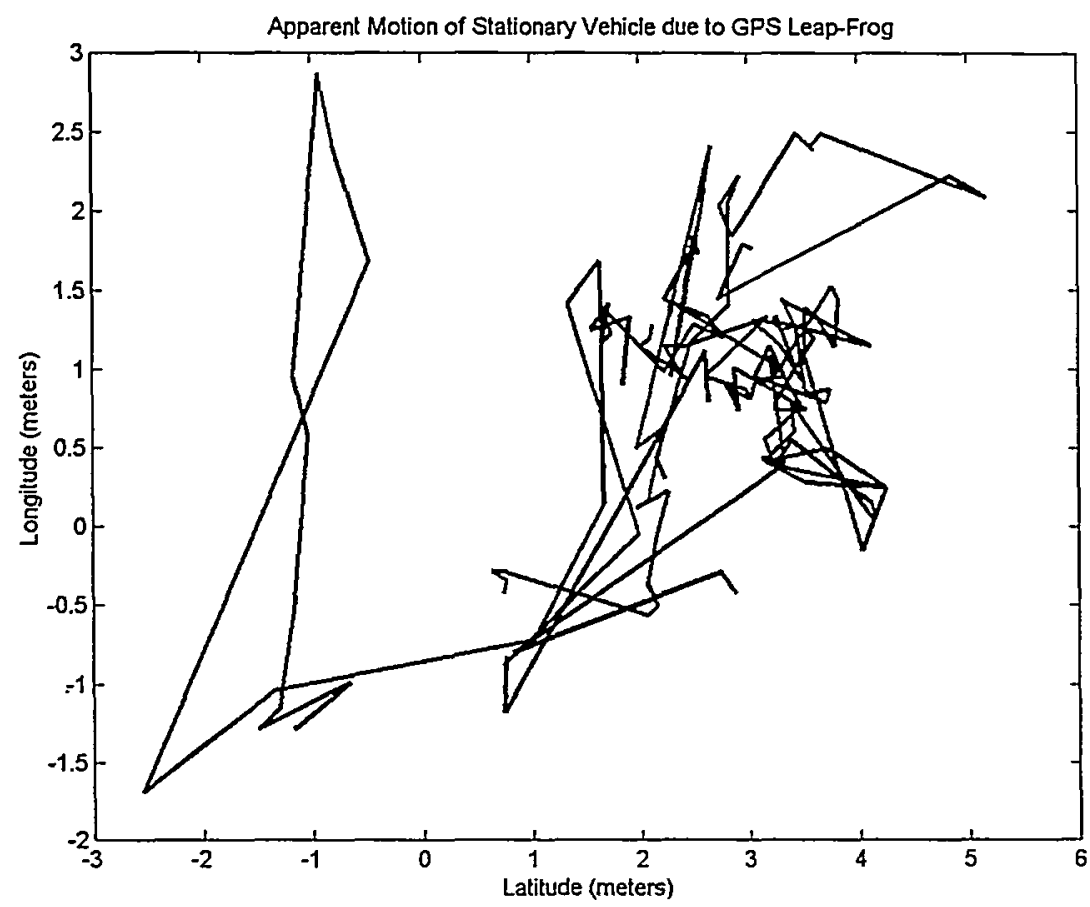

Figure 4 GPS errors accumulated by a stationary vehicle using corrections from a pair of mobile vehicles leapfrogging through a path with 12 exchanges.

\section{References}

1. D Bapna, E et .al., "The Atracama Desert Trek: Outcomes," Proceedings of the 1998 Conference on Robotics \& Automation, Leuven, Belgium, May $1998,597-604$.

2. Y. Uny Cao, Alex S. Fukunaga, and Andrew B. Kahng, "Cooperative Mobile Robotics: Antecedents and Directions," Proceedings of the 1995 IEEE/RSJ IROS Conference, 226-234.

3. T. Fukuda, et al, "Evaluation on Flexibility of Swarm Intelligent System, "Proceedings of the 1998 Conference on Robotics \& Automation, Leuven, Belgium, May 1998, 3210-3215.

4. B. Brummitt. and Anthony Stentz, "GRAMMPS: A Generalized Mission Planner for Multiple Mobile Robots In Unstructured Environments," Proceedings of the 1998 Conference on Robotics \& Automation, Leuven, Belgium, May 1998, 1564-1571. 
5. K. Kosuge, T. Oosumi, M. Satou, K. Chiba, K. Takeo, "Transporation of a Single Object by Two Decentralized-Controlled Nonholonomic Mobile Robots," Proceedings of the 1998 Conference on Robotics \& Automation, Leuven, Belgium, May 1998, 2989-2994.

6. Hiroaki Yamaguchi, "A Cooperative Hunting Behavior by Mobile Robot Troops," Proceedings of the 1998 Conference on Robotics \& Automation, Leuven, Belgium, May 1998, 3204-3209.

7. F.R. Noreils, "Multi-Robot Coordination for Battlefield Strategies," Proceedings of the 1992 IEEE.RSJ International Conference on Intelligent Robots and Systems, Raleigh, NC, July 1992, 1777-1784.

8. Q. Chen, J.Y.S. Luh, "Coordination and Control of a Group of Small Mobile Robots," Proceedings of the 1994 IEEE.RSJ International Conference on Intelligent Robots and Systems, 2315-2320.

9. J.P. Desai, J. Ostrowski, V. Kumar, "Controlling Formations of Multiple Mobile Robots," Proceedings of the 1998 Conference on Robotics \& Automatio $\mathrm{n}$, Leuven, Belgium, May 1998, 2864-2869.

10. H. Yamaguchi, J.W. Burdick, "Asymptotic Stabilization of Multiple Nonholonomic Mobile Robots Forming Group Formations," Proceedings of the 1998 Conference on Robotics \& Automation, Leuven, Belgium, May 1998, 3573-3580.

11. J.E. Hurtado, R.D. Robinett, C.R. Dohrmann, S.Y. Goldsmith, "Distributed Sensing and Cooperating Control for Swarms of Robotic Vehicles," Proc. IASTED Conference Control \& Applications, Honolulu, Hawaii, Aug. 12-14, 1998.

12. J.S. Jennings, G. Whelan, W.F. Evans, "Cooperative Search and Rescue with a Team of Mobile Robotis," Proc. IEEE International Conference of Advanced Robotics, Monterey, CA, 1997.

13. S. Goldsmith, J. Feddema, R. Robinett, "Analysis of Decentralized Variable Structure Control for Collective Search by Mobile Robots," SPIE98, Proc. Sensor Fusion and Decentralized Control in Robotic Systems, Boston, November 1-6, 1998.

14. C. Lewis, J.T. Feddema, P. Klarer, "Robotic Perimeter Detection System," Proceedings of SPIE Vol. 3577, Boston, MA, November 3-5, 1998, pp. 14-21.

15. J. Feddema, C. Lewis, P. Klarer, "Cooperative Robotic Sentry Vehicles," Proceedings of SPIE Vol. 3839, Boston, MA, September 19-20, 1999.

16. Bradford W. Parkinson and Per K. Enge, "Differential GPS," Global Positioning System: Theory and Applications, Volume II, Bradford W. Parkinson, James J. Spiker Jr., Editors, Progress in Astronautics and Aeronautics, Vol. 164., American Insitute of Aeronautics and Astronautics, Inc., 1996. 